University of South Florida

DIGITAL COMMONS

Digital Commons @ University of

@ UNIVERSITY OF SOUTH FLORIDA

South Florida

School of Geosciences Faculty and Staff

Publications

6-1988

\title{
Effect of Wet Tropospheric Path Delays on Estimation of Geodetic Baselines in the Gulf of California Using the Global Positioning System
}

\author{
David M. Tralli \\ Timothy H. Dixon \\ thd@usf.edu
}

Scott A. Stephens

Follow this and additional works at: https://digitalcommons.usf.edu/geo_facpub

Part of the Earth Sciences Commons

\section{Scholar Commons Citation}

Tralli, David M.; Dixon, Timothy H.; and Stephens, Scott A., "Effect of Wet Tropospheric Path Delays on Estimation of Geodetic Baselines in the Gulf of California Using the Global Positioning System" (1988). School of Geosciences Faculty and Staff Publications. 520.

https://digitalcommons.usf.edu/geo_facpub/520

This Article is brought to you for free and open access by the School of Geosciences at Digital Commons @ University of South Florida. It has been accepted for inclusion in School of Geosciences Faculty and Staff Publications by an authorized administrator of Digital Commons @ University of South Florida. For more information, please contact digitalcommons@usf.edu. 


\title{
Effect of Wet Tropospheric Path Delays on Estimation of Geodetic Baselines in the Gulf of California Using the Global Positioning System
}

\author{
David M. Tralli, Timothy H. Dixon, and Scott A. Stephens ${ }^{1}$ \\ Jet Propulsion Laboratory, California Institute of Technology, Pasadena
}

\begin{abstract}
Geodetic baseline measurements using the Global Positioning System (GPS) were acquired in the Gulf of Calıfornia between Loreto and Cabo San Lucas in Baja Californıa and Mazatlan on the mainland of Mexico. Tropospheric water vapor content was high during the experiment, typically yielding wet path delays in excess of $20 \mathrm{~cm}$ at zenith. Surface meteorological (SM) and water vapor radiometer (WVR) measurements were recorded at each site, providing independent means of calibrating the GPS signal for the wet tropospheric path delay. Residual wet delays at zenith are estimated as constants and also as first-order exponentially correlated stochastic processes. In addition, the entire wet zenith delay is estimated stochastically without prior calibration. The results of these approaches are compared in terms of day-to-day baseline repeatability and other system performance discriminants. Calibration with WVR data yields the best repeatabilities, of the order of 1-7.5 parts in $10^{8}$ in the horizontal components of 350to $650-\mathrm{km}$ baselines with carrier phase data. Further improvement in these results occurs if combined carrier phase and pseudorange data are used. WVR data are important for direct characterization of the wet tropospheric path delays in humid regions. SM measurements, if used with a simple atmospheric model and estımation of residual zenith delays as constants, can introduce significant errors in baseline solutions. However, SM calibration and stochastic estimation of residual zenith wet delays may be adequate for precise estimation of GPS baselines, with a deterioration in repeatability of less than 1-2 cm compared to WVR calibration. Stochastic estimation of the entire zenith wet delay yields comparable repeatabilities, particularly if both carrier phase and pseudorange data are used. Similar analyses of the Owens Valley Radio Observatory-Mojave baseline in California, where zenith wet delays are factors of 3-5 less, show no significant differences among the various tropospheric calıbrations.
\end{abstract}

\section{INTRODUCTION}

The first geodetic baseline measurements across the Gulf of California (Mexico) using radio signals from the Global Positioning System (GPS) satellites were acquired in November 1985 through a cooperative effort by many institutions under the direction of the Jet Propulsion Laboratory (JPL), sponsored by the National Aeronautics and Space Administration [Dixon et al., 1988]. The experiment was designed to assess the feasibility and potential accuracy of GPS-based geodesy in the gulf and to establish epoch measurements for part of a future network. Continued baseline measurements in the region will constrain kinematic models of this part of the Pacific-North America plate boundary.

The sites occupied in this experiment include Loreto and Cabo San Lucas in Baja California and Mazatlan on the mainland of Mexico, in addition to several "fiducial" stations in the United States (Figure 1). Since this study reports on the first occupation in the Gulf of California, no GPS-based spreading rates can be determined. However, an assessment of the precision and potential accuracy of these geodetic measurements can be made. In this paper, strategies for estimating and correcting the effects of tropospheric water vapor are evaluated. Tropospheric path delays can be a major source of error in space-based geodetic techniques [Dravskikh and Finkelstein, 1979], such as very long baseline interferometry (VLBI) [e.g., Kroger et al., 1986; Herring, 1986; Treuhaft and Lanyi, 1987]. The gulf experiment is significant in that tropospheric water vapor content was high, with wet path delays regularly exceeding $20 \mathrm{~cm}$ at zenith. Water vapor radiometer

${ }^{1}$ Now at University of Illinois, Urbana.

Copyright 1988 by the American Geophysical Union.

Paper number 7B2063.

0148-0227/88/007B-2063\$05.00
(WVR) measurements were taken at each site, providing an opportunity to evaluate their effectiveness for tropospheric calibration. If the troposphere represents the largest contribution to system noise, it is expected that WVRs will yield better results relative to alternate calibration techniques, based on experience with VLBI and covariance studies [Kroger et al., 1986], as well as Seasat altimeter data, which yielded improved height measurements when microwave radiometers were used for calibration [Tapley et al., 1982].

In this study, the efficacy of WVR calibration of the wet tropospheric path delay is rigorously assessed relative to calibration based on surface meteorological (SM) measurements and to stochastic estimation of the entire wet delay without prior calibration. Once representative values for stochastic parameters describing the wet troposphere in a given region and season are obtained, stochastic estimation may prove an economical alternative to the acquisition of WVR data in future GPS experiments or may allow greater flexibility in the disposition of limited numbers of WVRs among several sites. The analyses are undertaken using only carrier phase data (integrated Doppler) and also using combined carrier phase and pseudorange data. Residual zenith wet path delays, after SM or WVR calibration, are estimated simultaneously with the geodetic parameters at each site as constant offsets or biases spanning the satellite observation periods and alternately as first-order exponentially correlated stochastic processes. Baseline repeatabilities, differences in the mean baseline solutions, consistency of the wet troposphere solutions, and comparison of solutions on a VLBI baseline in southern California provide discriminants by which to evaluate the various wet tropospheric calibration schemes.

\section{Tropospheric Path Delay}

\section{Background}

Radio wave propagation in the troposphere is nondispersive, and therefore path delays cannot be calibrated by obser- 


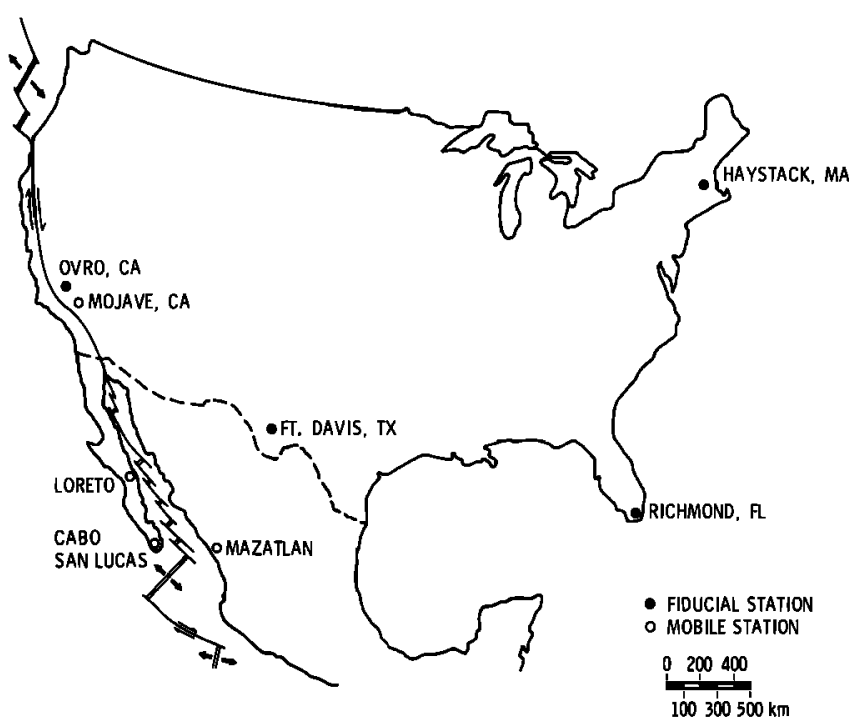

Fig. 1. Approximate locations of sites occupied in this study. The Pacific-North America plate boundary is highly simplified.

vations at different frequencies [e.g., Spilker, 1978]. The troposphere contains about $80 \%$ of atmospheric mass and nearly all water vapor and clouds [Gill, 1982]. It is also characterized by convective heat transfer, implying the possibility of azimuthal asymmetry. Estimation of tropospheric path delays involves either direct measurement, measurement of other parameters which are related through simple physical or empirical models, or the use of statistical or probabilistic models which fit the spatial and temporal characteristics of the troposphere. A review of the various methods is given by Nahvi et al. [1986].

In this study, estimation of the GPS signal delay introduced by propagation through the wet troposphere is undertaken in three ways: (1) use of SM measurements combined with a simple atmospheric model, (2) direct measurements of atmospheric water vapor content using WVRs, and (3) stochastic estimation according to the apparent temporal behavior of the wet troposphere. Although statistical characterization of the troposphere could be based on historical data or previously developed models, the best indicator of short-term tropospheric behavior comes from actual WVR data recorded during the experiment, recognizing that this may be dependent on site, season, and weather.

Surface meteorological measurements. Surface measurements of atmospheric temperature, barometric pressure, and relative humidity can be combined with simple atmospheric models to obtain estimates of the tropospheric path delay at zenith. The relations are briefly summarized in this section.

The Fermat measured path length $L_{e}$ of a radio wave which propagates along a path $L$ through the atmosphere is given by

$$
L_{e}=\int_{L} n(s) d s
$$

where $n(s)$ is the refractive index as a function of position $s$ along the path $L . L_{e}$ is also known as the apparent electrical length. The difference between $L_{e}$ and the straight-line geometrical distance (slant range) $G$ is the excess path length:

$$
\Delta L=L_{e}-G=\int_{L}[n(s)-1] d s+(S-G)
$$

where $S$ is the path length along $L . \Delta L$ includes propagation delays and a contribution due to refractive bending [Dodson, 1986]. The $S-G$ term is insignificant, about $1 \mathrm{~cm}$ or less at elevation angles greater than about $15^{\circ}$ [Resch, 1984], although it could easily be modeled using ray-tracing techniques. Clearly, the effects of ray bending and horizontal inhomogeneities are more important for slant paths; for observations at zenith, $S$ is equal to $G$.

The atmosphere may be modeled simply as spherically symmetric shells. Model refractivity profiles can be derived empirically from radiosonde measurements of atmospheric parameters at various heights. The path delay itself may be expressed as an empirical function of meteorological measurements. For example, Smith and Weintraub [1953] give the refractivity $N$, defined by

$$
N=(n-1) \times 10^{6}
$$

as a function of total atmospheric pressure $\boldsymbol{P}$ (in millibars), atmospheric temperature $T$ (in kelvins), and partial water vapor pressure $e$ (in millibars):

$$
N=77.6(P / T)+3.73 \times 10^{5}\left(e / T^{2}\right)
$$

This expression is considered accurate to $0.5 \%$ for frequencies less than $30 \mathrm{GHz}$ in normal ranges of $P, T$, and relative humidity [Resch et al., 1985]. The two terms in equation (4) are usually referred to as the "dry" and "wet" components, respectively. Generally, about $90 \%$ of the total refractivity is attributable to the dry component.

The wet troposphere component is less uniform both spatially and temporally relative to the dry component. It is also more likely to be mismodeled, since the distribution of liquid water and vapor is much less predictable than that of temperature and pressure [Chao, 1973]. Hogg et al. [1981] used radiosonde profiles to measure the short-term ( $2 \mathrm{~min}$ to 1 day) temporal spectrum of precipitable water vapor and showed that to within about $1 \%$ accuracy the wet path delay is 6.5 times the total precipitable water vapor; however, the correlation with surface humidity is highly variable according to site and season [Reber and Swope, 1972].

The dry troposphere refractivity is integrated by assuming hydrostatic equilibrium combined with an equation of state for dry air which relates barometric pressure, temperature, and air density. The corresponding dry path delay is about 220$230 \mathrm{~cm}$ at zenith. Moisture effects on density are not usually significant [Gill, 1982]. However, horizontal gradients in the atmospheric temperature at a given elevation can cause gradients in density even if the surface pressure is independent of position [Bender, 1983]. Horizontal gradients in the dry component may occur due to mountain lee winds but in general are thought to be small. Bender [1983] suggests that horizontal variations might be estimated if SM data are taken at various sites in the vicinity of the region of interest [see Gardner et al., 1978; Gibbs and Majer, 1981].

The dry troposphere model used in this study is a linear function of surface pressure, assuming static equilibrium and the ideal gas law. If these conditions are satisfied, the uncertainty in the zenith dry delay is $\pm 2 \mathrm{~mm}$ given a precision of \pm 1 mbar in the surface barometric pressure measurement [Hopfield, 1971]. The zenith dry path delay $\Delta \rho_{z_{\mathrm{dry}}}$ (in meters) is given by

$$
\Delta \rho_{z \mathrm{dry}}=2.276 P_{0}
$$


where $P_{0}$ is the surface pressure (in bars). In the absence of a pressure measurement a nominal pressure for a station at elevation $h$ (in meters) above mean sea level is

$$
P_{0} \approx 1.013 e^{-h / H}
$$

where 1.013 bars is sea level pressure and $H$ is the scale height with an adopted value of $7000 \mathrm{~m}$ [Bean and Dutton, 1966].

The expression used in this study for predicting the zenith wet delay $\Delta \rho_{z_{\text {wet }}}$ (in meters) from surface meteorological measurements is given by Chao [1974]:

$$
\Delta \rho_{z_{\mathrm{wCl}}}=\chi\left[1.63 \frac{e_{\mathrm{o}}{ }^{1.23}}{T_{K}{ }^{2}}+2.05 \gamma \frac{e_{0}{ }^{146}}{T_{K}{ }^{3}}\right]
$$

where $\gamma$ is the temperature lapse rate (in kelvins per kilometer) and $e_{0}$ is the surface vapor pressure (in newtons per square meter)

$$
e_{0}=6.1(\mathrm{RH}) 10^{(A T /(B+T))}
$$

$T_{K}=T+273.15$ and $\mathrm{RH}$ is the (percent) surface relative humidity. The coefficient $A$ equals 7.4475 , and $B$ equals $234.7^{\circ} \mathrm{C}$, with temperature $T$ (in degrees Celsius) measured at the surface. The multiplicative scaling factor $\alpha$ depends on site altitude and is related to the model derivation from radiosonde balloon data [Chao, 1974], confining the wet refractivity profiles to the first $6.1-7.6 \mathrm{~km}$ of altitude, where most of the atmospheric water vapor is contained [Chao, 1973]. Sur[ace measurements may not always be dependable, a point which will be discussed later. For example, inconsistencies in surface measurements of temperature or relative humidity can occur due to diurnal effects (especially differential ground heating) and the presence of a thin layer of high humidity near the surface. Thus there may not be a direct relation between total atmospheric water content and surface meterological measurements.

Water vapor radiometers. Wet tropospheric path delays can also be estimated with microwave radiometer measurements. WVRs measure the microwave radiation emitted by water vapor in the atmosphere. The technique is described by Westwater [1967, 1978], Clafin et al. [1978], Wu [1979], Resch et al. [1982, 1985], Janssen [1985], Gary et al. [1985], and S. E. Robinson (unpublished manuscript, 1987).

Briefly, a WVR yields measurements of the brightness temperature of the atmosphere, which can be related to the amount of precipitable vapor and liquid through the equation of radiative transfer [e.g., Chandrasekhar, 1950]. Measurements are taken near a spectral line at $22.235 \mathrm{GHz}$, corresponding to a resonance in the water molecule. Measurements at one or two additional frequencies are necessary to separate the effects of vapor and liquid and pressure broadening [e.g., Resch et al., 1985, Figure 6-1]. "Tipping curves" (elevation scans) are used to minimize instrumental error by calibrating with respect to the cosmic blackbody temperature and the expected elevation angle dependence of a homogeneous atmosphere [Claflin et al., 1978]. Equations are formulated to solve for the excess path delay due to water vapor and the integrated liquid content in the atmosphere in terms of apparent brightness temperature [Resch, 1984; Resch et al., 1985].

WVR measurements may be made by copointing along the line of sight to the radio source (e.g., GPS satellite). Effects attributable to azimuthal variations in water vapor content are then incorporated into the measurements. More commonly, however, repeated tipping curves are performed to obtain a good estimate of the average zenith delay, implicitly assuming azimuthal symmetry. Examples of azimuthal variations in tropospheric water content, which tend to map into horizontal baseline components [Bender, 1983], are given by Hargrave and Shaw [1978] and Coco and Clynch [1982]. Nevertheless, azimuthal variations are typically small, less than $2 \mathrm{~cm}$ of path delay (M. A. Janssen, personal communication, 1987). The data from this experiment suggest negligible azimuthal variation.

The model which combines WVR and meteorological data to derive a wet tropospheric path delay is known as the "retrieval algorithm." In the past, the retrieval algorithm has been based on radiosonde data which provide profiles of atmospheric parameters that can be used to calibrate the microwave brightness temperature measurement. Since the path delay is a site-dependent function of temperature, pressure, and water vapor density, a statistical analysis of measured brightness temperatures versus radiosonde-measured delay is undertaken. There are uncertainties, however, due to limitations in the accuracy of the radiosonde sensors [Yang et al., 1981]. The retrieval algorithm may also introduce systematic errors due to site and seasonal variations in addition to the instrument calibration uncertainties [Resch, 1984; Resch et al., 1985; Beckman, 1985]. Covariance analyses of GPS system accuracy have suggested an improvement by up to a factor of 2 with the use of WVR data, provided the instrumental noise is below about $0.5 \mathrm{~cm}$. Absolute calibration of WVRs remains incomplete, but direct calibration by comparison with independent measurements is planned [Bender, 1983; Walter and Bender, 1987]. Without such calibration the expected uncertainty of a WVR-based zenith delay is about 1-2 cm (rms) [Davidson and Trask, 1985].

Stochastic modeling. Spatial and temporal water vapor fluctuations in the atmosphere, or equivalently variations in refractivity, can be modeled statistically or characterized by probabilistic laws. This approach was used by Treuhaft and Lanyi [1987] to estimate the effect of tropospheric delays in VLBI measurements. An advantage of a stochastic formulation is that spatial and temporal variations of water vapor can be described mathematically and to some extent predicted over varying spatial dimensions and time scales.

In this study, the time-dependent behavior of the wet tropospheric path delay is modeled as a first-order Gauss-Markov process, which is defined by the differential equation

$$
d p / d t=-p(t) / \tau_{p}+w(t)
$$

where $p$ is the model parameter corresponding to the zenith wet delay, $\tau_{p}$ is the correlation time of the process, and $w(t)$ is a zero-mean white noise random variable of variance $\sigma_{w}{ }^{2}$ given by the ensemble average of its square

$$
\left\langle w(t) w\left(t^{\prime}\right)\right\rangle=\sigma_{w}^{2} \delta\left(t-t^{\prime}\right)
$$

where angle brackets denote the expectation value operator and $\delta\left(t-t^{\prime}\right)$ is the Dirac delta function. The discrete solution to equation (9) is

$$
p(t+\Delta t)=m p(t)+\left(1-m^{2}\right)^{1 / 2} w_{p}
$$

where

$$
m=e^{\left(-\Delta t / \tau_{p}\right)}
$$

is a measure of the (exponential) time correlation between adjacent measurements. If $\tau_{p}$ is much shorter than the measurement interval $\Delta t$, adjacent measurements become un- 
correlated. The steady state deviation of the process $\sigma_{p}$ is determined from

$$
\sigma_{p}^{2}=\left\langle w_{p}^{2}\right\rangle
$$

in the limit of time $t$ greater than the correlation time $\tau_{p}$ and is related to the correlation time by

$$
\sigma_{p}^{2}=\tau_{p} \sigma_{w}{ }^{2} / 2
$$

A derivation of these expressions is provided by Bierman [1977].

Modeled as a first-order Gauss-Markov stochastic process, the wet tropospheric path delay at zenith can be parameterized by two values, $\tau_{p}$ and $\sigma_{p}$. The quantity $m$ may be obtained from the ensemble average of the covariance,

$$
\begin{aligned}
& \langle p(t+\Delta t) p(t)\rangle=\left\langle m p^{2}(t)\right\rangle+\left\langle\left(1-m^{2}\right)^{1 / 2} p(t) w_{p}\right\rangle \\
& \langle p(t+\Delta t) p(t)\rangle=m\left\langle p^{2}(t)\right\rangle
\end{aligned}
$$

given that $\left\langle w_{p}\right\rangle$ equals zero. Equation (12) then allows $\tau_{p}$ to be expressed as a function of process measurements (through $m$ ) and measurement interval $\Delta t$. Similarly, an expression for the steady state deviation $\sigma_{p}$ can be derived using equations (11) and (12). Values of $\tau_{p}$ obtained in this study (Table 2) range from 0.2 to 10 hours and in most cases are less than half the satellite observation periods, while measurement intervals are $6 \mathrm{~min}$. This indicates that steady state behavior will typically be evidenced within a given observation period (about 4-8 hours) and that adjacent data points are highly correlated. In the limit of an infinite correlation time and infinite steady state variance a Markov process approaches a random walk, the stochastic model used in other studies [e.g., Lichten and Border, 1987]. The two formulations are nevertheless similar.

In the formalism proposed here, the process $p$ is the zenith wet tropospheric path delay, for example, derived from actual WVR measurements during satellite observation periods at each site or from residual zenith delays after calibration. Stochastic estimation is carried out simultaneously with estimation of the geodetic parameters of interest and yields the entire wet zenith delay (with no prior calibration) or a residual zenith delay (after SM or WVR calibration). In the former case, values of the stochastic parameters $\tau_{p}$ and $\sigma_{p}$ may be obtained directly from the WVR-based zenith delays, while in the latter case, appropriate values are determined empirically. Both approaches are considered in this study. Stochastic estimation of residual delays has the advantage that potential errors in the calibration can be modeled. Stochastic estimation without prior calibration, using values of $\tau_{p}$ and $\sigma_{p}$ which span those based on the WVR data, is also undertaken to assess the sensitivity of baseline solutions to these parameter values. To some extent this indicates the marginal utility of WVRs in terms of relative improvements over other calibration techniques in the absence of WVRs.

\section{Elevation Angle Mapping Function}

Tropospheric path delays depend on the geometrical path length through the troposphere (equation (2)) and are therefore functions of the elevation angle from the ground receiver to the radio source (e.g., GPS satellite). The estimate of the delay appropriate at a given elevation angle can be based on the value at zenith obtained from WVR or SM measurements, combined with simple atmospheric models [e.g., Chao, 1974]. Alternately, the WVR measurements and copointing retrieval algorithms can be used directly, as discussed above.
The tropospheric delay $\tau_{\text {Irop }}$ is expressed as a linear combination of the dry and wet components:

$$
\tau_{\text {trop }}=\rho_{z_{\mathrm{dry}}} R_{\mathrm{dry}}(E)+\rho_{z_{\mathrm{wel}}} R_{\mathrm{wel}}(E)
$$

where $\rho_{z}$ terms are zenith delays and $R$ is an elevation angle mapping function. $E$ is the apparent elevation angle to the observed radio source. The analytic elevation angle mapping function used in this study is that developed by Lanyi [1984], which is accurate to the level of $1 \%$ at a $6^{\circ}$ elevation angle. Others are given by Berman [1970], Saastamoinen [1972], Chao [1973], and Davis et al. [1985]. The functional form of the tropospheric delay is

$$
\tau_{\text {trop }}=F(E) / \sin E
$$

where $F(E)$ is a function of wet and dry zenith delays and path-bending delays, atmospheric scale height and average surface temperature (which determines the scale height), mean molecular mass of dry air, and gravitational acceleration. The path delays are expressed as moments of wet and dry refractivities [Lanyi, 1984].

Errors in the mapping function, particularly at low elevation angles, can be a major error source in estimating the path delay. Model inadequacies related to the assumption of no horizontal gradients in atmospheric parameters and errors in the actual surface measurements can also lead to incorrect path delay estimates [Dodson, 1986]. Horizontal refractivity gradients cause deviations from the simple cosecant of the elevation angle scaling and may introduce up to $3-\mathrm{cm}(\mathrm{rms})$ delay errors at elevation angles of about $10^{\circ}$ [Gardner, 1976]. Horizontal pressure gradients and inadequacies in the dry troposphere mapping functions may result in a systematic error of about $1 \mathrm{~cm}$ in the vertical baseline component [Davidson and Trask, 1985].

\section{Estimation of Geodetic Parameters}

The application of GPS for high-precision geodesy is discussed by Bossler et al. [1980], Remondi [1985], Bock et al. [1986b], Beutler et al. [1987], Lichten and Border [1987], and others. A typical GPS experiment consists of a network of receivers at "mobile" and "fiducial" sites, with measurements recorded simultaneously during periods of mutual satellite visibility among all stations in the network, resulting in about 4-8 hours of data per day for a span of a few days. The positions of the mobile sites are determined by multiparameter estimation methods. Fiducial sites (fixed) are used to define a terrestrial reference frame, as well as to constrain satellite orbital parameters. One may also choose to treat a subset of the fiducial network as mobile sites for comparison of their location solutions to known VLBI-based values and thus obtain some measure of system performance and accuracy. In this study, baseline component solutions are obtained separately for each day of data and then combined to yield mean values. The scatter is the day-to-day repeatability and is a measure of precision.

The analytical method employed here involves simultaneous estimation of satellite trajectories, satellite and receiver clock biases, carrier phase ambiguities, mobile receiver positions, and tropospheric delays at zenith (or residual zenith delays if independent calibration is available) and allows treatments of dynamic Earth model parameters and satellite radiation parameters. The GPS inferred positioning system (GIPSY) software is used, developed at JPL and described by 
TABLE 1. Wet Troposphere Calıbrations Applied in This Study

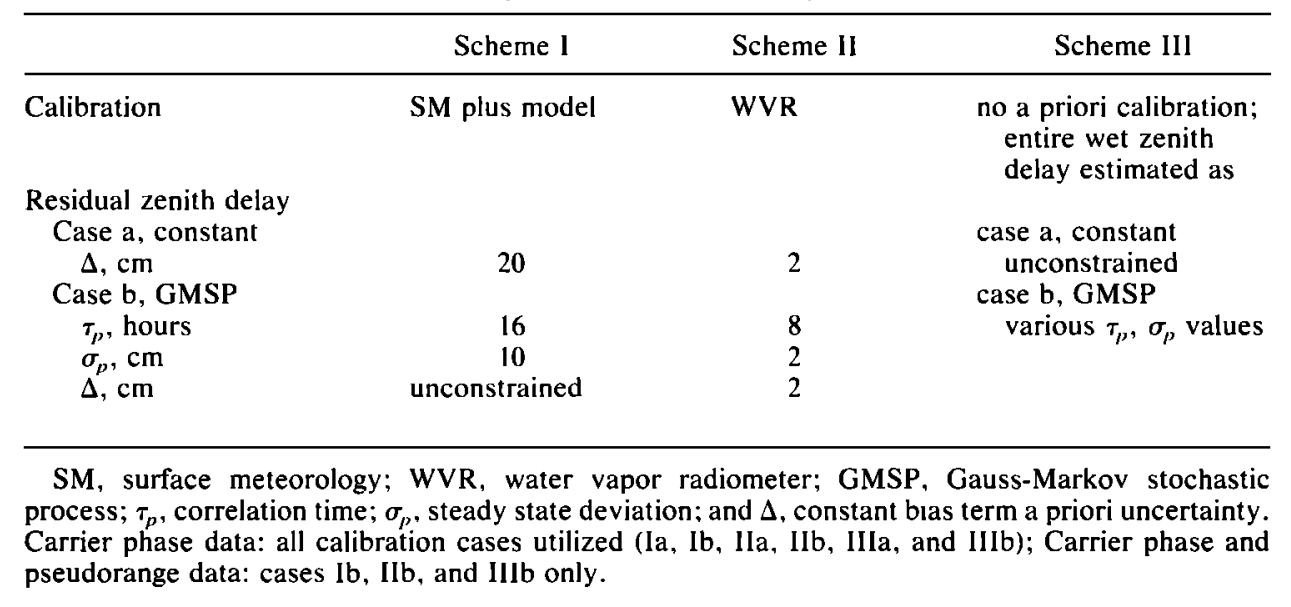

Wu et al. [1986], Davidson et al. [1987], and Lichten and Border [1987]. The parameter estimation algorithm is a batch sequential $U-D$ filter [Bierman, 1977; Thornton and Bierman, 1980], similar to a Kalman filter [Kalman, 1960] but providing greater numerical stability. Satellite and receiver clocks are modeled as white noise processes with a reference clock at a given receiver, equivalent to double differencing [e.g., Bock et al., 1986a]. A satellite elevation angle cutoff of $15^{\circ}$ is used to minimize the effects of multipathing and atmospheric propagation errors. The data interval is $6 \mathrm{~min}$ after compression. Owing to the lengths of the baselines and the correspondingly large differential ionospheric effects, bias fixing is not attempted; rather, the range ambiguity is estimated jointly with the other parameters.

\section{Data}

The November 1985 GPS experiment spanned a 13-day period, 10 days of which yielded data for one or more of the sites at Loreto and Cabo San Lucas in Baja California and Mazatlan on the mainland of Mexico [Dixon et al., 1988]. Equipment problems at one or more sites limited high-quality data at all three sites in the Gulf of California to 6 consecutive days (November 18-23). Problems with both the carrier phase and pseudorange data at Loreto on November 20 and 21 were noted. Analyses of postfit residuals indicated larger rms scatter (by a factor of about 2) at this site, possibly related to calibration problems in the receiver and increased data noise. Inclusion of these data yielded baseline solution outliers which dominated the repeatability regardless of tropospheric calibration. Data from Loreto on November 20 and 21 are therefore not used in this study. Figure 1 shows the gulf sites relative to the entire U.S. fiducial network. The longest baseline across the gulf is approximately $650 \mathrm{~km}$, from Loreto to Mazatlan; Loreto to Cabo San Lucas is about $450 \mathrm{~km}$; and Mazatlan to Cabo San Lucas is about $350 \mathrm{~km}$. The fiducial network consists of international radio interferometric surveying (IRIS) sites (Haystack, Massachusetts; Fort Davis, Texas; and Richmond, Florida) and the Owens Valley Radio Observatory (OVRO) in California. GPS data are also used from a VLBI site at the Deep Space Network facility at Mojave, California, which is treated as a mobile site in order to establish a control baseline to OVRO and to provide some measure of system accuracy. Results for the gulf baselines based on various fiducial network configurations are reported by Dixon et al. [1988].
Carrier phase data are used, along with pseudorange data from all sites where available (TI-4100 receivers). SERIES-X receivers were deployed at OVRO and Mojave, and only carrier phase data were obtained at these sites. The time span of data collected at each gulf site is indicated in Table 2 . Seven GPS satellites were available (NAVSTARS 3, 4, 6, 8, 9, 10, 11), although the amount of data from satellite 11 is small. The WVR used at Cabo San Lucas is a new three-channel model (J-01) [Janssen, 1985], while the WVRs at the other two gulf sites are older two-channel units (R0-4 [Resch et al., 1985] at Mazatlan and SCAM at Loreto). All sites recorded SM data consisting of temperature, barometeric pressure, and relative humidity at approximately half-hour intervals. No WVR data were available for Mazatlan on two of the days. In addition, review of the field operator logs indicated rain on November 22 at Cabo San Lucas and light rain on November 21 at Cabo San Lucas, on November 22 at Mazatlan, and on November 23 at Loreto. Liquid water in the atmosphere hampers WVR performance. In addition, a thin film of water can form near the horn of the WVR antenna. Unless this film is removed prior to a measurement, the WVR data are degraded as the presence of near-field liquid water contributes significant thermal radiation into the measurement beam. Initially, these WVR data were used and yielded inconsistencies in wet troposphere and baseline solutions which suggested erroneous calibrations. Although WVR calibrations for several site days were suspect, only the WVR data on November 22 at Cabo San Lucas and on November 23 at Loreto introduced significant variation from otherwise normal distributions of baseline solutions, which are particularly sensitive to outliers given the small population size. WVR data from these two site days are therefore not used.

\section{Calibration of Wet Tropospheric Path Delays}

Three approaches to calibrating the GPS signal for wet tropospheric path delays are presented (summarized in Table 1): scheme I, calibration with SM data combined with a simple atmospheric model [e.g., Chao, 1974]; scheme II, calibration with WVR data; and scheme III, no a priori calibration, but estimation of the entire wet zenith delay along with the geodetic parameters of interest. In schemes I and II, residual zenith delays are estimated for case a as constant over the satellite observation period at each station with a given a priori uncertainty, or for case $b$ as a first-order exponentially 
TABLE 2. Correlation Times $\tau_{p}$ and Steady State Deviations $\sigma_{p}$ Obtained from WVR Tipping Curves Over Indicated Data Spans for Gulf of California Sites

\begin{tabular}{|c|c|c|c|}
\hline Day & $\begin{array}{c}\text { Data Span, } \\
\text { hours }\end{array}$ & $\tau_{p}$, hours & $\sigma_{p}, \mathrm{~cm}$ \\
\hline \multicolumn{4}{|c|}{ Loreto } \\
\hline 18 & 5.8 & 9.57 & 1.72 \\
\hline 19 & 6.5 & 2.96 & 0.78 \\
\hline 20 & 6.1 & 2.81 & 0.51 \\
\hline 21 & 6.5 & 5.75 & 1.09 \\
\hline 22 & 1.9 & 0.29 & 0.22 \\
\hline 23 & 6.9 & 0.54 & 0.36 \\
\hline \multicolumn{4}{|c|}{ Mazatlan } \\
\hline 18 & 4.5 & 7.25 & 1.73 \\
\hline 19 & 6.5 & 6.55 & 2.10 \\
\hline 20 & 6.2 & 0.60 & 0.91 \\
\hline 21 & 6.4 & 0.25 & 0.53 \\
\hline 22 & 6.2 & 1.28 & 1.40 \\
\hline 23 & 5.9 & 0.29 & 0.43 \\
\hline \multicolumn{4}{|c|}{ Cabo San Lucas } \\
\hline 18 & 8.4 & 5.81 & 0.76 \\
\hline 19 & 7.2 & 0.19 & 0.34 \\
\hline 20 & 8.5 & 1.17 & 0.95 \\
\hline 21 & 8.4 & 0.55 & 0.38 \\
\hline 22 & 7.7 & 0.00 & 0.95 \\
\hline 23 & 7.7 & 2.08 & 0.83 \\
\hline
\end{tabular}

Values for Mazatlan on days 18 and 19 are determıned from SM measurements and the Chao [1974] atmospheric model. A $\tau_{p}$ value of zero indicates a negative correlation (see equation (15)).

correlated (Gauss-Markov) stochastic process with characteristic correlation times $\tau_{p}$ and steady state deviations $\sigma_{p}$ determined empirically. For scheme III the entire wet zenith delay is estimated for case a as a constant or for case b as a stochastic process as described above. Various $\tau_{p}$ and $\sigma_{p}$ values are tested for case IIIb, in part derived from the WVR data (Table 2). On site days for which there are no WVR data, or if the WVR data appeared to be invalid as noted above, calibration for scheme II is instead based on SM measurements with subsequent stochastic estimation of the residual zenith delay (as in case Ib).

All six cases described above are tested with the carrier phase data type. In addition, a subset of these (the more precise stochastic cases Ib, IIb, and IIIb) are tested using the more robust combination of carrier phase and pseudorange data. Note that modeling the zenith delay as a zero-mean Markov-type stochastic process requires joint estimation of a constant offset term. This allows some assessment of differences in repeatability due solely to modeling tropospheric fluctuations by comparing case a to case b solutions. For simplicity, the various calibration schemes are applied only to the three gulf sites, since these have the largest wet path delays. Tropospheric calibration at the remaining sites uses WVRs if available (case IIa) (i.e., at OVRO and Mojave) or SM measurements and the Chao [1974] atmospheric model (case Ia). However, in an attempt to achieve the best possible precision, one network analysis is performed with optimized tropospheric calibration at all sites with requisite stochastic parameters determined empirically from initial analyses.

Since the functional form of the mapping function for both wet and dry tropospheric components is similar it is difficult to estimate simultaneously both wet and dry zenith path delays. Therefore only the zenith wet delay is estimated; the dry tropospheric calibration is assumed correct, considered only as a potential systematic error at the level of $1 \mathrm{~cm}$ [Davidson and Trask, 1985]. The time scale of dry variations tends to be much longer and of the order of one-third the wet variation [Treuhaft and Lanyi, 1987]. For this experiment the wet troposphere at the gulf sites is also very stable over periods of hours.

Except for instrumental uncertainties, errors associated with the retrieval algorithm, and potential azimuthal asymmetries, the WVR data yield a direct estimate of the delay due to tropospheric water vapor. Stochastic estimation of a residual delay after WVR calibration (case IIb) would ideally include error models for the WVR. This is not attempted specifically in this study. The values of the stochastic parameters used for estimation of residual zenith delays after WVR calibration (case IIb), namely, 8-hour correlation time and 2-cm steady state deviation (Table 1), are determined empirically and are consistent with the level of fluctuations in WVR-based zenith delays. Stochastic estimation of zenith residuals after SM calibration (case Ib) similarly should incorporate errors in the empirical atmospheric models. However, as demonstrated in the following section, atmospheric models constrained only by SM measurements are intrinsically not very dependable due to both highly localized atmospheric variations and instrumental uncertainties, neither of which is amenable to accurate quantitative characterization at this time. A correlation time of 16 hours and a steady state deviation of $10 \mathrm{~cm}$ for SM calibrated data (Table 1) are also empirically determined and are representative of the scatter noted in the SM data. Correlation times of 8 and 24 hours and steady state deviations of 6 and $20 \mathrm{~cm}$ were also tested.

\section{Discussion of Results}

\section{Assessment of Tropospheric Calibrations for the Gulf Sites}

A comparison of the repeatabilities obtained for the three baselines in the Gulf of California using the various wet tropospheric calibration methods (Table 1) is shown in Figure 2 using carrier phase data only and in Figure 7 with combined carrier phase and pseudorange data. The histograms show the root-mean-square (rms) scatter about the means of the components obtained from each of the single-day solutions, weighted by the corresponding standard errors. Note that baseline length is redundant with the horizontal components (east and north) and that for the mainly east-west Mazatlan to Cabo San Lucas baseline the east and length components are essentially identical. The largest uncertainty is typically in the east component when only carrier phase data are used. Several geometrical considerations, including the mainly north-south satellite ground tracks and satellite visibility and distribution, account for this lack of longitudinal resolving power. However, when pseudorange data are combined with carrier phase data, repeatability in the east component is greatly improved by constraining system clocks and range ambiguities. The vertical component repeatability then becomes the most weakly resolved but is always better than 1 part in $10^{7}$ (Figure 7).

Figure 2 shows that with carrier phase data only baseline repeatability is poor when SM data are used for calibration and the residual wet delay at zenith is estimated simply as a constant (case Ia). A significant improvement in horizontal baseline repeatability occurs if the residual wet delay is estimated instead as an exponentially correlated stochastic process (case Ib). Curiously, baseline repeatabilities, when the entire wet delay is estimated as a constant without prior cali- 


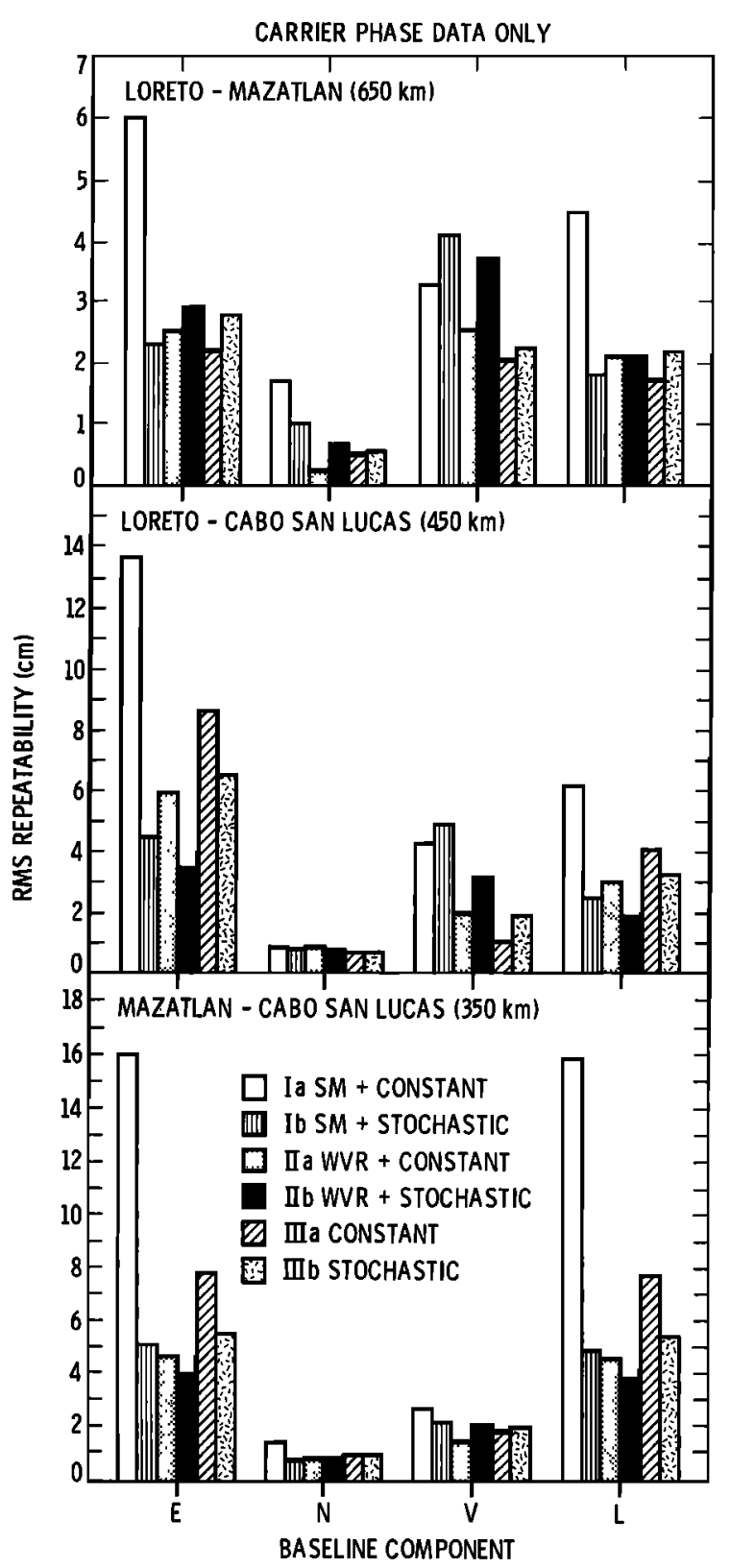

Fig. 2. Histogram of baseline component $(E, N, V)$ and length $(L)$ repeatabilities for the three Gulf of California baselines determined from the standard deviation about the weighted mean of each of the solutions. Only carrier phase data are used. The wet tropospheric calibration schemes are outlined in Table 1.

bration (case IIIa), are better than in case Ia. This indicates that simple SM calibration actually introduces errors into the baseline estimates. The effect of these SM errors is minimized if the residual zenith delay is allowed to be time varying (case Ib). Stochastic estimation of the entire wet zenith delay yields similarly good results (case IIIb). Some explanation for this behavior comes from the actual wet delays, as estimated from SM and WVR calibration. Figure $3 a$ shows a greater level of fluctuations in SM-based zenith delays relative to WVR-based delays. In addition, Figure $3 b$ indicates a significant difference between the two wet zenith delays. This offset may be attributable to the fact that all three gulf sites lie on the coast and marine layer conditions were present in the early morning (local time). A marine layer tends to homogenize temperature

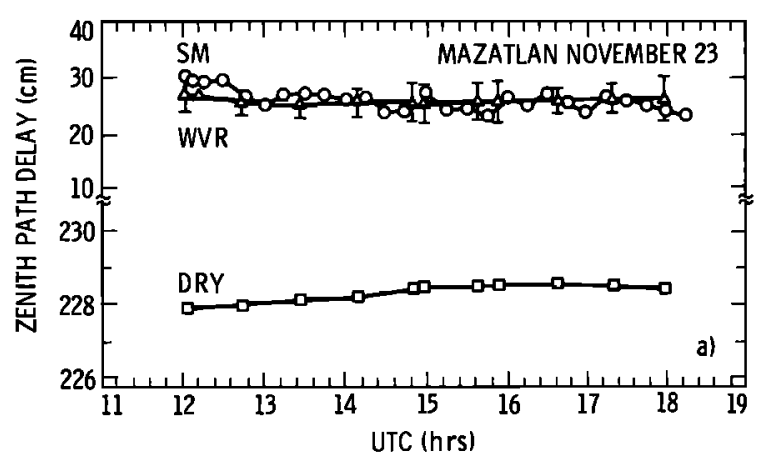

Fig. 3a. Wet tropospheric path delay at zenith for Mazatlan on November 23 based on WVR tipping curves (triangles) and SM measurements in conjunction with the Chao [1974] atmospheric model (circles). Error bars on the tipping curve are about $\pm 2 \mathrm{~cm}$, reflecting a known $4.5^{\circ}$ offset in the WVR beam direction at this site (S. E. Robinson, personal communication, 1987). Note the stable nature of the WVR-based zenith delays. Dry tropospheric path delays at zenith are also shown.

differences, and the troposphere behaves very stably. There will be a lack of correlation between surface measurements and tropospheric water content if simple atmospheric models are used which treat the temperature profile as linearly decreasing with altitude [e.g., Chao, 1973]. Since a marine layer creates an inversion in the actual temperature profile [see Dixon et al., 1988], zenith wet path delays based only on SM measurements can be in error by large amounts. Estimation of only a constant residual zenith delay does not adequately account for these errors in simple SM-based calibrations.

The baseline repeatabilities when only carrier phase data are used appear to be optimized with WVR calibration and stochastic estimation of residual zenith wet path delays (case IIb). The differences with case Ib are not dramatic, the largest being an improvement by $2.2-3.3$ parts in $10^{8}$ in the east component repeatabilities of baselines involving Cabo San Lucas and by 3.9 parts in $10^{8}$ in the vertical repeatability of the Loreto to Cabo San Lucas baseline; the Loreto to Mazatlan east repeatabilities actually become worse by about 1 part in $10^{8}$. The relatively greater improvement with WVR calibration for baselines involving Cabo San Lucas may reflect the use of the newer, three-channel J-01 WVR [Janssen, 1985] at this site. The day-to-day east component repeatabilities for case IIb are 4.5 parts in $10^{8}$ to 1.1 parts in $10^{7}$ for the three

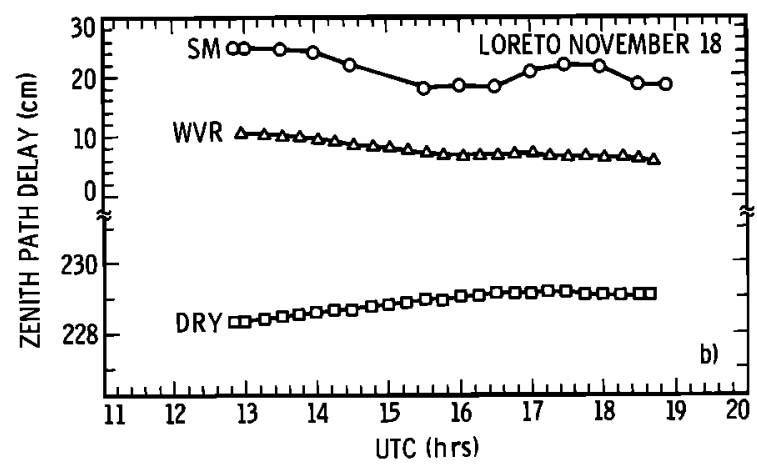

Fig. 3b. Wet tropospheric path delay at zenith for Loreto on November 18 (see caption Figure $3 a$ ). Note the stable nature of the WVR-based zentth delays. The offset between the SM- and WVRbased zenith delays is attributable to marine layer conditions (see text). Dry tropospheric path delays at zenith are also shown. 


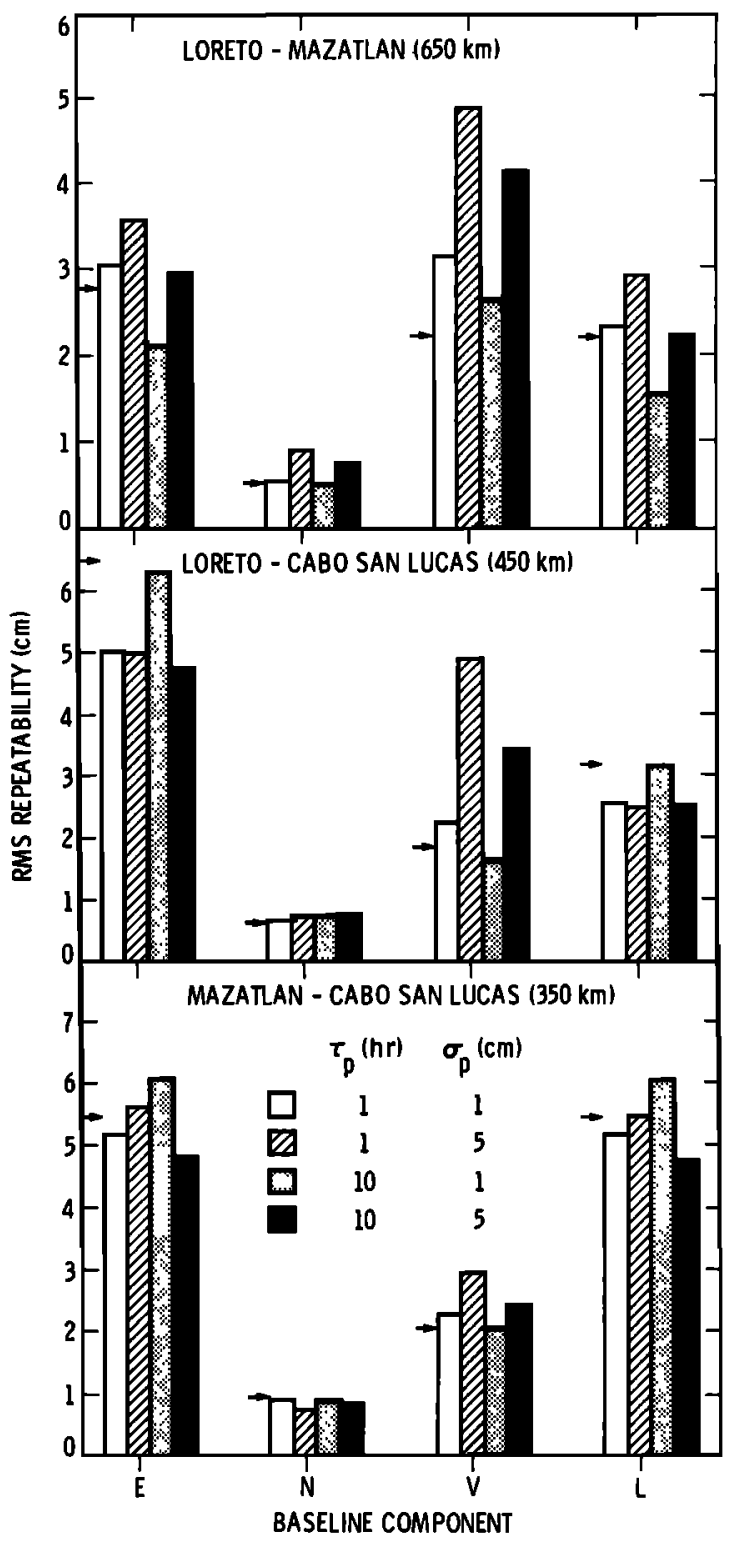

Fig. 4. Histogram of baseline component $(E, N, V)$ and length $(L)$ repeatabilities for case IIIb, and stochastic estimation of wet tropospheric zenith delay without prior calibration for values of $\tau_{p}$ and $\sigma_{p}$ of 1 and 10 hours and 1 and $5 \mathrm{~cm}$, respectively. Only carrier phase data are used. Arrows indicate repeatabilities obtained with stochastic parameter values determined from actual WVR-based zenith delays (Table 2 and Figure 2).

baselines, while north repeatabilities are $1.0-2.6$ parts in $10^{8}$. Vertical component repeatabilities range from 5.7 to 7.0 parts in $10^{8}$.

The agreement between cases IIIa and IIIb is surprising but consistent with the small magnitude (generally less than $2 \mathrm{~cm}$ and often less than $1 \mathrm{~cm}$ ) of the steady state deviations obtained from the WVR data (Table 2). Comparing the results from these two cases gives an indication of the improvement attributable to stochastic modeling. In order to test the sensitivity of case IIIb to the values of the stochastic parameters, baseline solutions were obtained for values of $\tau_{p}$ (1 and 10 hours) and $\sigma_{p}(1$ and $5 \mathrm{~cm})$ which span those obtained from the WVR data. These are compared to solutions using values listed in Table 2 (Figure 4). Although this is not an exhaustive analysis, it appears that baseline repeatabilities are more sensi- tive to the choice of steady state deviation $\left(\sigma_{p}\right)$ than correlation time $\left(\tau_{p}\right)$ for the range of tested values. The relatively small values of $\sigma_{p}$ characterizing the tropospheric delay during this experiment place rather tight constraints on the stochastic models. It is clear that there is some benefit to having the WVR data even if the troposphere is modeled stochastically, because optimum values of $\tau_{p}$ and $\sigma_{p}$ thereby can be obtained. What is important, and at this point unknown, is the longterm validity of such a stochastic parameterization for a given site.

The consistency of the various tropospheric calibrations can be investigated by considering the daily mean zenith wet tropospheric delay solutions. These are plotted in Figure 5 for each gulf site over the time span of the experiment. Generally, there is good agreement, with values tending to the mean zenith delays determined from WVR rather than SM calibration. Although mean solutions for Cabo San Lucas on November 20 are distinctly different (about $8 \mathrm{~cm}$ ) for cases Ia and Ib, residual zenith delays estimated stochastically (Ib) show variations of more than $\pm 5 \mathrm{~cm}$ (Figure 6), indicating that modeling of fluctuations can change significantly the estimate of the constant term. The uncertainties in the estimated con-

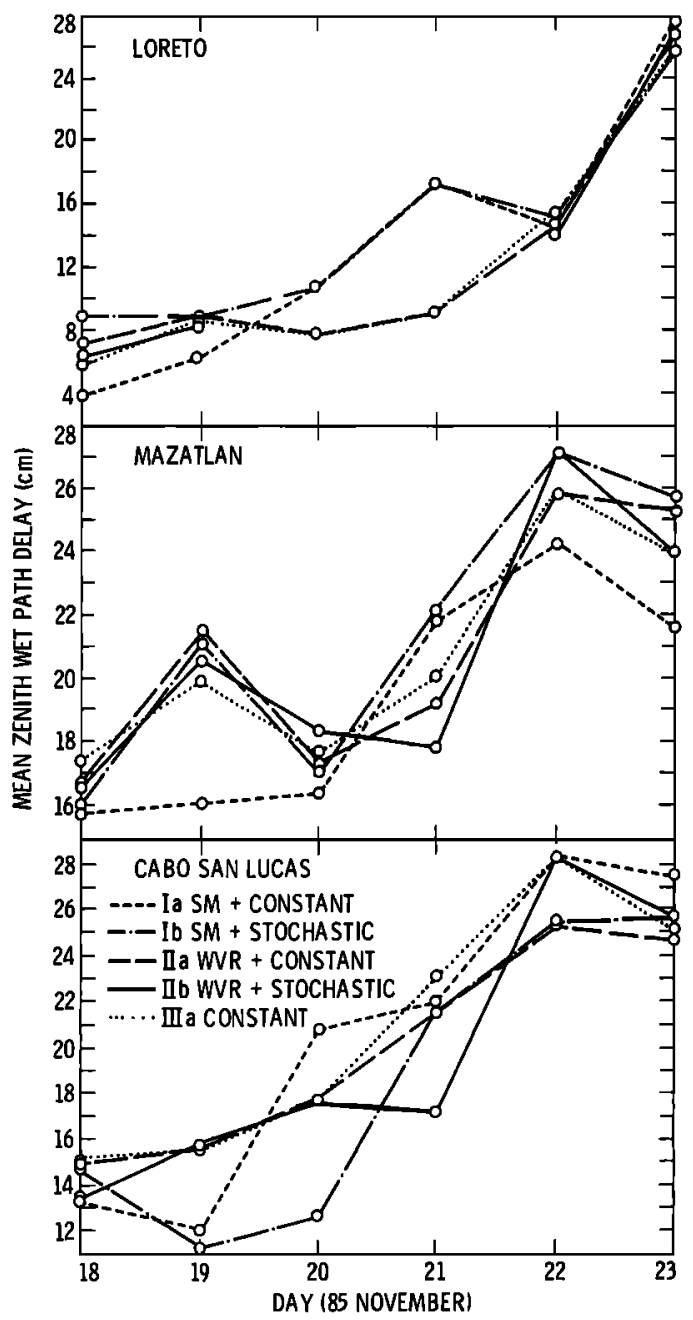

Fig. 5. Mean zenith wet tropospheric delay solutions for each gulf site according to the indicated calibration schemes using only carrier phase data. Mean zenth delays from case IIIb are omitted for clarity but fall within $1 \mathrm{~cm}$ of the case IIIa values. For Loreto on November 20 and 21 (not used in the analyses), SM- and WVR-based mean zenith delays are shown for continuity. 


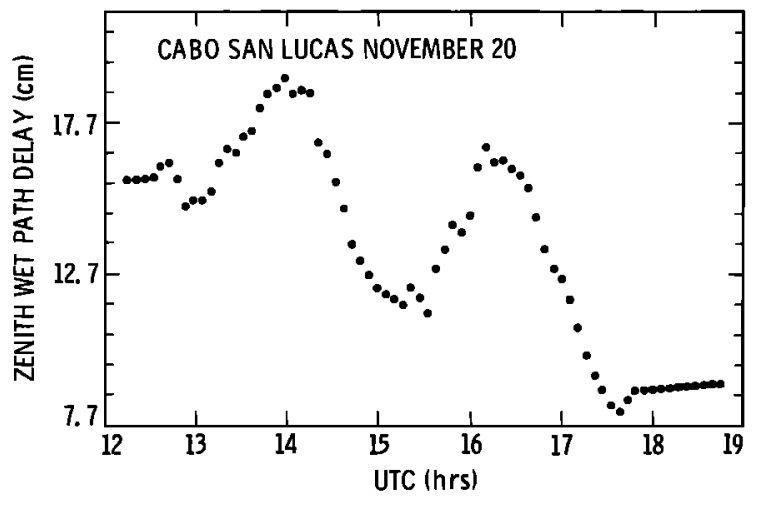

Fig. 6. Stochastic estimation solution of residual zenith wet tropospheric path delay at Cabo San Lucas on November 20 using case Ib, SM calibration, and stochastic estimation of residual wet zenith delay. Values are superimposed on a constant equal to the sum of the mean of the SM calibration $(26.28 \mathrm{~cm})$ and the estımated offset $(-13.57 \mathrm{~cm})$.

stant terms are $1-2 \mathrm{~cm}$ in all cases except cases $\mathrm{Ib}$ and IIb where the uncertainties are about $10 \mathrm{~cm}$, comparable to the steady state deviations. While Figure 5 shows that wet zenith delays increased over the 6 days of the experiment, the troposphere was stable over any given 4 - to 8 -hour satellite observation period.

Figure 7 shows the baseline component repeatabilities for solutions based on combined carrier phase and pseudorange data, with SM and WVR calibration and stochastic estimation of residual zenith wet delays (cases Ib and IIb, respectively, and with stochastic estimation of the entire wet zenith delay without prior calibration (case IIIb). Pseudorange data better define system timing offsets and constrain carrier phase range ambiguities. Uncertainties in these parameters tend to map mainly into the east baseline component. Clock errors are reflected in the east component due to the geometry of satellite trajectories and estimation of station positions on a rotating Earth. With the addition of pseudorange data the east component repeatabilities improve by up to a factor of 5 relative to values using only carrier phase data. For the Loreto to Cabo San Lucas baseline, east repeatability improves from 7.5 to 2 parts in $10^{8}$ (case IIb) and from 1.4 parts in $10^{7}$ to 2.8 parts in $10^{8}$ (case IIIb); for the Mazatlan to Cabo San Lucas baseline, improvement is from 1.1 parts in $10^{7}$ to 3.8 parts in $10^{8}$ (case IIb) and from 1.55 parts in $10^{7}$ to 3.8 parts in $10^{8}$ (case IIIb). The Loreto to Mazatlan baseline shows no change. Case Ib shows similar improvements, except for Mazatlan to Cabo San Lucas where the east repeatability improves by almost a factor of 2 (from 1.45 parts in $10^{7}$ to 8.4 parts in $10^{8}$ ). North component repeatabilities show improvements by factors of 1.5-2 for the Loreto to Cabo San Lucas and Mazatlan to Cabo San Lucas baselines for cases Ib and IIb. For case IIb the north repeatability for Loreto to Mazatlan improves by a factor of about 3 , but the vertical gets slightly worse. North component repeatabilities for case IIIb show up to a factor of 2 improvement.

The vertical component becomes the most poorly determined when combined carrier phase and pseudorange data are used (Figure 7). This is similar to high-precision VLBIdetermined baselines, where tropospheric effects are also the dominant error source and map mainly into the vertical component [e.g., Kroger et al., 1986]. If only carrier phase data are used, east repeatabilities are most poorly determined (Figure
2). Wet tropospheric calibration based on WVR data and estimation of a residual zenith delay as a correlated stochastic process (case IIb) improves the vertical repeatabilities by a few parts in $10^{8}$, or about a factor of 2 for the $450 \mathrm{~km}$ Loreto to Cabo San Lucas baseline, compared to calibration based on SM (case Ib), and only slightly for the Loreto to Mazatlan baseline $(650 \mathrm{~km})$. The improvements in repeatability attributable to WVR calibration appear significant for both the east and vertical baseline components. Although the east repeatabilities are improved overall with the addition of pseudorange data, the relative differences between WVR and SM calibration generally remain the same. However, with combined carrier phase and pseudorange data, stochastic estimation of the entire wet zenith delay without prior calibration (case IIIb) yields vertical component repeatabilities which are better than

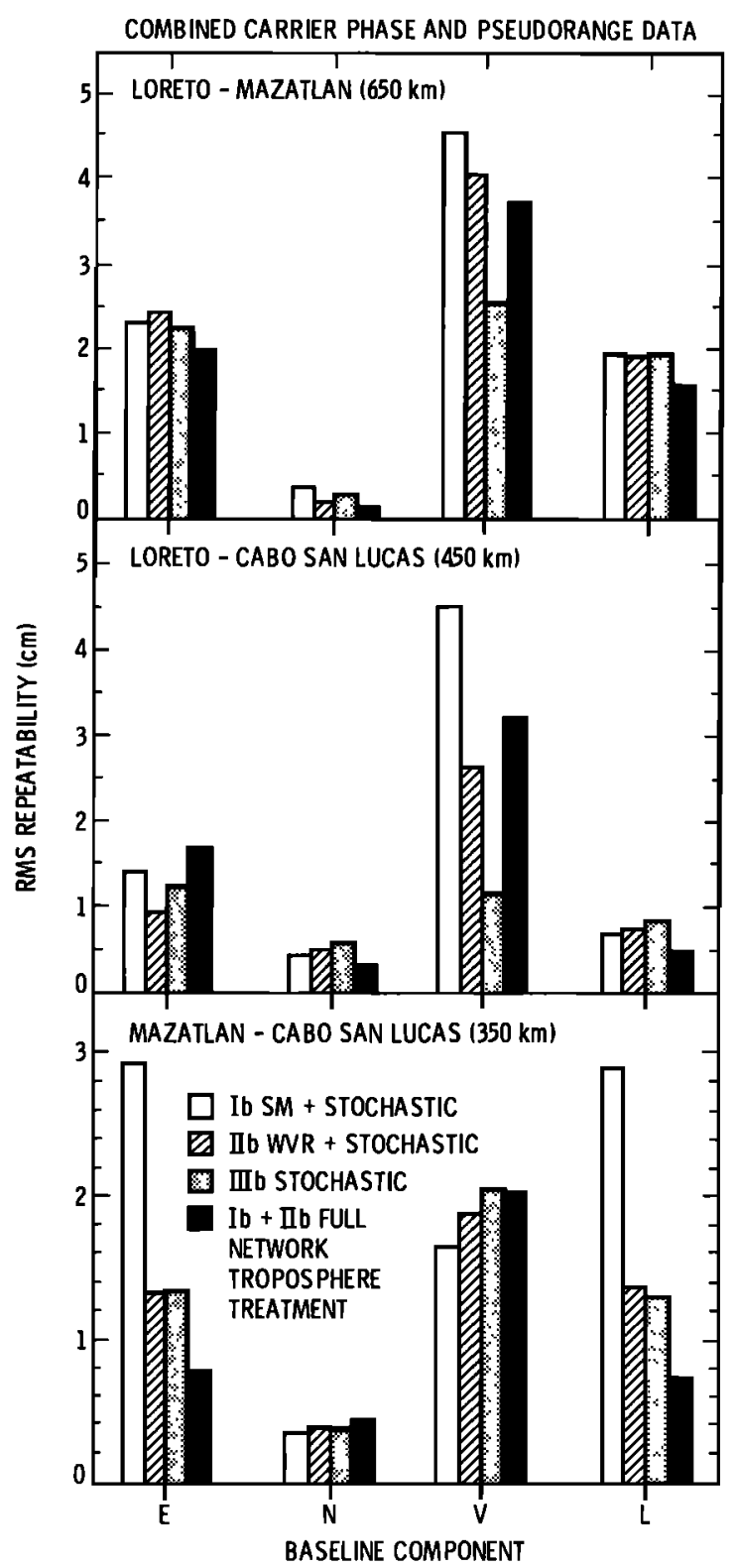

Fig. 7. Histogram of baseline component $(E, N, V)$ and length $(L)$ repeatabilitıes for each gulf baselıne using combined carrier phase and pseudorange data and the more precise estimation schemes Ib, IIb, and IIIb (see Table 1). A full network analysis, where case Ib or IIb is used at every site (rather than just the guln) as dictated by the availability of WVRs, is also shown for comparison. 

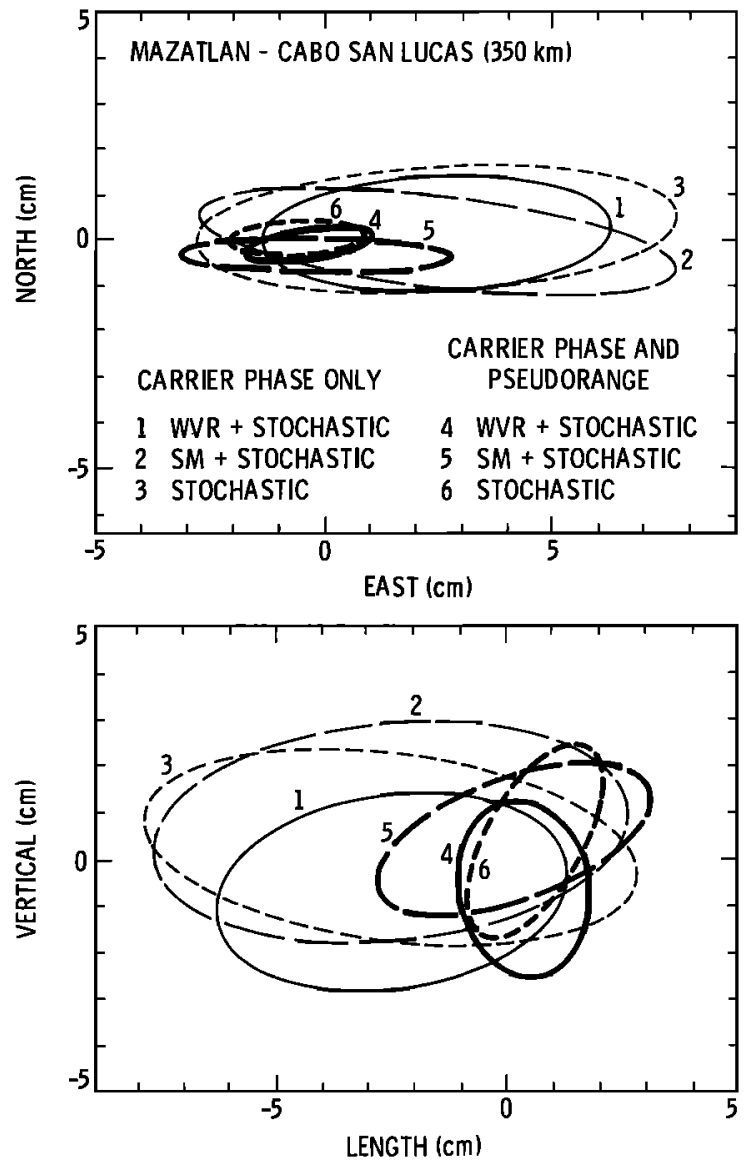

Fig. 8. Mazatlan to Cabo San Lucas (350 km) baseline solutions for cases Ib, IIb, and IIIb using only carrier phase data and combined with pseudorange data. Ellipses denote day-to-day repeatabilities for 6 days of data, plotted about the corresponding weighted means (see text).

those obtained with WVR calibration (by factors of about 1.5 and 2 for the Loreto to Mazatlan and Loreto to Cabo San Lucas baselines, respectively). Horizontal repeatabilities are roughly equivalent. Given the high level of precision of a few parts in $10^{8}$ achievable with combined carrier phase and pseudorange data [e.g., Lichten and Border, 1987], it may be that errors in the WVR calibration and instrumental noise become the dominant error sources. Further stochastic analyses of residual delays may allow these errors to be modeled.

Throughout the study, data below $15^{\circ}$ in satellite elevation angle have been excluded, as noted previously. GPS measurements to lower elevations may provide better estimates of tropospheric delays because of greater sensitivity to the elevation angle function (i.e., longer propagation paths through the troposphere), as suggested by experience with VLBI. To assess this, a subset of baseline solutions using only carrier phase data were obtained including data down to a $5^{\circ}$ elevation angle. Results based only on cases Ia and IIIb (Table 1) indicated that although uncertainties (standard errors) in baseline components decrease by a few millimeters due to the additional data, day-to-day repeatability is significantly worsened (by several centimeters), particularly in the east component. This is attributable to multipath effects. These effects would be worse for pseudorange data because of the higher sensitivity of this data type to multipathing [e.g., Evans, 1986]. It is tentatively concluded that low elevation data do not yield improved troposphere estimates at this time, presumably because the low-gain, omnidirectional receiver antennae used in this experiment are susceptible to multipath and below-thehorizon refraction. This differs from VLBI, where highly directional antennae are used.

\section{System Precision and Accuracy}

In order to assess the maximum achievable precision attributable to correcting for tropospheric effects the optimum tropospheric calibrations demonstrated by this study are applied to the entire eight station network. Thus WVR calibration is used where possible, SM calibration is used elsewhere, and residual zenith delays at each station are estimated stochastically with correlation times of 8 or 16 hours and 2- or 10-cm steady state deviations, respectively, depending on whether WVR or SM data are used for calibration (Table 1). Both carrier phase and pseudorange data are used where available. These results are shown in Figure 7. The baseline repeatabilities for east, north, and vertical components range from 2.2 to 3.8 parts in $10^{8}, 1$ part in $10^{9}$ to 1.2 parts in $10^{8}$, and 5.6 to 7 parts in $10^{8}$, respectively. These are improved relative to case IIb, where the optimum (WVR) calibration was applied only at the gulf sites. However, no effort was undertaken to optimize values of the stochastic parameters for the U.S. sites. Furthermore, only a subset of the data have high-quality WVR measurements available (section 4), suggesting that further improvements in system precision and accuracy might be possible if high-quality WVR data were available at all humid sites (including Richmond, Florida).

In addition to day-to-day repeatability, it is desirable to assess the accuracy of the gulf baselines. Of course, this is not possible rigorously without a well-established regional reference frame and independent measurements. Site markers connecting the GPS and satellite laser ranging (SLR) monuments at Cabo San Lucas and Mazatlan have not yet been surveyed. However, it is useful to look at potential systematic shifts in baseline solutions resulting from the various analytical methods. A comparison of Mazatlan to Cabo San Lucas mean baseline solutions for cases Ib, IIb, and IIIb (see Table 1), using only carrier phase data and combined with pseudorange data, is shown in Figure 8. These results are typical of the other gulf baselines. The error ellipses are one standard deviation repeatabilities about the mean solution for each approach, weighted by the corresponding errors of each daily solution, and are plotted about the mean of all cases shown. The agreement among the baseline solutions from each approach is consistent with the previously noted agreement in the mean zenith wet path delay solutions (Figure 5). The addition of pseudorange introduces a shift of about $3 \mathrm{~cm}$ in the east component solution. The significance of this will only become clearer with future accuracy assessments and comparison to SLR data. However, this shift is within the uncertainties of the present solutions, and it is not unexpected given the better definition of system timing offsets with pseudorange data. The various methods agree at about 1 part in $10^{7}$.

The baseline from OVRO (fiducial) to Mojave (mobile), included in all the gulf baseline analyses, also allows for assessment of system accuracy since VLBI data are available for comparison. Estimates of this baseline ensures, to some extent, that there are no systematic errors in the analytical approach and that differences in the gulf baseline repeatabilities are a valid discriminant of the performance of a particular tropospheric calibration. Note that the OVRO-Mojave baseline re- 


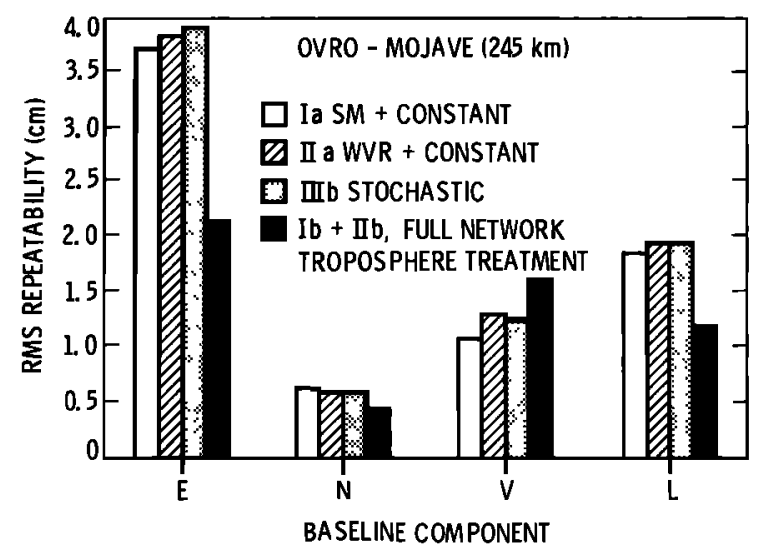

Fig. 9. Histogram of baseline component $(E, N, V)$ and length $(L)$ repeatabilities for the OVRO-Mojave baseline for SM and WVR calibration (cases Ia and IIa) and stochastic estimation (case IIIb). Only carrier phase data are used, with a five-station network as described in text (i.e., Baja sites excluded). Repeatabilities with a full network (i.e., Baja sites included) tropospheric calibration, using cases Ib and Ilb and pseudorange data where available, are also shown.

peatabilities are actually slightly worse if the gulf sites are excluded (Figure 9). Inclusion of gulf sites for OVRO-Mojave baseline estimates may strengthen network geometry and enhance control of satellite orbital parameters even if the three gulf sites are treated as mobile (S. L. Lichten, personal communication, 1987). The agreement of these OVRO-Mojave baseline solutions with VLBI is within about $3 \mathrm{~cm}$, similar to other results [Bock et al., 1986b]. This suggests a system accuracy of about 1 part in $10^{7}$ in southern California. Since the precision achieved on the OVRO-Mojave baseline (several parts in $10^{8}$ for horizontal components) is comparable to that achieved in the Gulf of California, we suggest that the accuracy of gulf baselines is also about 1 part in $10^{7}$.

\section{Wet Tropospheric Calibration for Drier Sites}

A specific study of the OVRO-Mojave baseline was undertaken, with the gulf sites excluded, in order to test the various wet tropospheric calibration methods in a dry environment. The wet delay at zenith here is about $5-10 \mathrm{~cm}$, much less than the values of up to $8-28 \mathrm{~cm}$ observed in the Gulf of California (Figure 5). IRIS stations and OVRO were used as fiducials, and Mojave was treated as mobile. Only carrier phase data were used. Furthermore, only the tropospheric calibration at OVRO was varied because WVR data at Mojave are limited

TABLE 3. Correlation Times $\tau_{p}$ and Steady State Deviations $\sigma_{p}$ Determined From WVR Tipping Curves Over Periods of About 24 Hours for OVRO, California

\begin{tabular}{lcc}
\hline Day & $\tau_{p}$, hours & $\sigma_{p}, \mathrm{~cm}$ \\
\hline 13 & 8.59 & 0.59 \\
14 & 10.25 & 0.67 \\
15 & 5.32 & 0.37 \\
16 & 3.55 & 0.63 \\
18 & 13.94 & 0.54 \\
19 & 40.46 & 1.12 \\
20 & 22.15 & 1.53 \\
21 & 37.27 & 2.20 \\
22 & 23.05 & 1.08 \\
23 & 19.79 & 0.97 \\
24 & 2.87 & 0.78 \\
\hline
\end{tabular}

Days correspond to November 1985. to 3 days [Dixon et al., 1988]. The correlation times and steady state deviations obtained from WVR tipping curves for 11 days of data at OVRO are given in Table 3. Although steady state deviations of $1-2 \mathrm{~cm}$ are comparable to those for gulf sites, the correlation times (particularly from November 18-23) are longer, factors of 2-6 times the satellite observation periods. These values were obtained from approximately 24hour periods of WVR data that are representative of any given observation period. The results of the analyses are shown in Figure 9 in terms of baseline repeatability and show agreement to within $0.25 \mathrm{~cm}$. Figure 9 also shows OVROMojave repeatabilities obtained from applying optimal tropospheric calibrations to the entire network, as previously discussed. The baseline length repeatability is then about 5 parts in $10^{8}$. Recall, however, that pseudorange data are not available at these two sites.

SM- and WVR-based zenith delays at OVRO for 1 day are shown in Figure 10. The agreement is representative of any given day. A figure similar to Figure 5 for the site at OVRO would show an increase from about 2 to $8 \mathrm{~cm}$ in the mean wet path delay at zenith over the 11-day observation period, with most values less than $5 \mathrm{~cm}$. The tropospheric water content is much less than at the gulf sites, and the level of fluctuations in the zenith wet path delay is similarly small. Even when deviations reach $1-2 \mathrm{~cm}$, the correlation times are sufficiently large (typically exceeding 10 hours, Table 3 ) that these values will not be evidenced in the span of a typical GPS observation session. It appears that the troposphere for these southern California locations can be adequately calibrated with SM measurements combined with stochastic models, at least at the current level of system accuracy and assuming conditions similar to this experiment. More analyses will be required to verify this, however. Thus WVR data may not be necessary in drier regions provided weather conditions are not severe.

\section{Discussion of $W V R$ Utility}

WVR data for tropospheric path delay calibration in humid regions appear to be important for achieving optimum precision and accuracy in GPS-based geodetic baseline measurements and for quantitatively characterizing the wet tropospheric path delay. However, WVRs may not be necessary in dry regions. Stochastic methods for tropospheric calibration may be an attractive alternative to WVR calibration even at

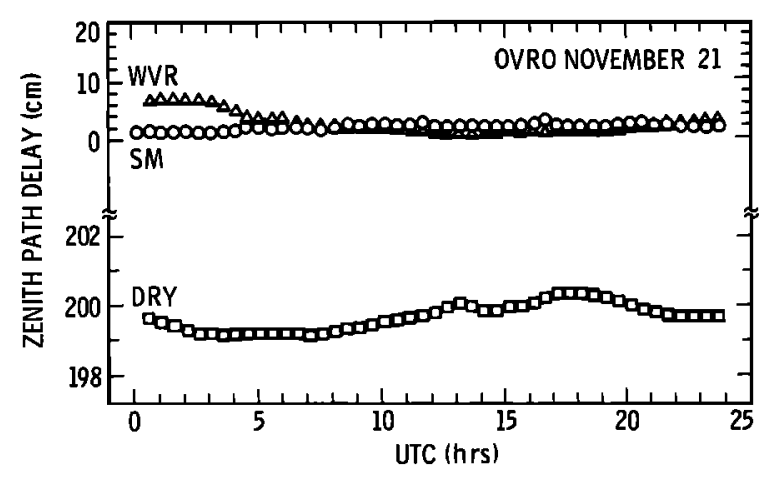

Fig. 10. Zenith wet tropospheric delay at OVRO on November 21 determined from SM measurements, the Chao [1974] atmospheric model (circles), and WVR tipping curves (triangles). Values are usually less than $5 \mathrm{~cm}$, in contrast to Gulf of California sites (Figure 3). Note the agreement between the WVR- and SM-based values, in contrast to Figure 3. Dry zenith tropospheric delays are also shown. 
humid sites, at least for tropospheric conditions similar to those encountered in this experiment and particularly with combined use of carrier phase and pseudorange data.

Two main factors limit the generality of these conclusions and suggest the need for further studies. First, during this experiment the wet troposphere at the gulf sites was remarkably stable over periods of satellite visibility (4-8 hours). This is indicated by the WVR data, the ability to model the wet path delay at zenith simply as a constant at each site, and the overall agreement of the various zenith wet path delay solutions with the mean WVR-based values. Thus the relatively simple atmospheric and stochastic models used in this study can adequately characterize tropospheric effects. WVRs may prove more important in highly variable weather. Second, the WVR data used in this study were not optimum. Older twochannel WVRs were used at two of the gulf sites and may be more susceptible to systematic errors relative to the newer three-channel unit used at Cabo San Lucas (B. L. Gary, personal communication, 1987). The older units are also laboratory systems which were not designed for field use, resulting in lack of data on some days. In addition, WVR data were not available at the Richmond, Florida, fiducial site, which is located in a humid environment. Studies of baseline repeatability in the southern Gulf of California, as a function of fiducial network configuration, indicate that Richmond is a critical fiducial site, presumably because of the added geometric extent of the network [Dixon et al., 1988]. For these reasons, it is likely that tropospheric calibration with WVRs can be improved significantly relative to this experiment. Since tropospheric calibration may be the limiting error source when pseudorange data are available (yielding precision of a few parts in $10^{8}$ ), it appears that further improvements in GPS system performance are achievable, even in humid environments, if optimum WVR data are available.

A rigorous assessment of WVR utility in GPS geodesy requires data precision of the order of several parts in $10^{8}$ or better, depending on tropospheric water content. Otherwise, studies of WVR utility will yield ambiguous results if tropospheric water vapor contents are low or if data reduction techniques are not sufficiently precise to detect correspondingly small tropospheric calibration errors. In such cases, tropospheric errors may not be the limiting error, and other sources (e.g., orbit uncertainties) will likely dominate the error budget. In general, the appropriate treatment of the wet troposphere will depend on the region of interest, on the stability of the troposphere or level of tropospheric fluctuations, and on whether pseudorange data are used in conjunction with carrier phase data.

\section{Conclusions}

In a humid region such as the Gulf of California, where the wet path delay at zenith may exceed $20 \mathrm{~cm}$, WVR data are important for characterization of the wet troposphere and precise estimation of GPS baselines. Day-to-day horizontal baseline component repeatability of a few parts in $10^{8}$ is demonstrated using WVR calibration and stochastic estimation of the residual zenith delay. The baseline accuracy appears to be about 1 part in $10^{7}$ based on the internal consistency of baseline solutions using different tropospheric calibration schemes and comparison of the OVRO-Mojave baseline in southern California with VLBI solutions.

SM measurements for wet tropospheric calibration can in- troduce significant errors in baseline solutions, but these are mitigated if residual zenith delays are modeled stochastically. However, stochastic estimation of the entire zenith wet tropospheric delay without prior calibration also yields good baseline repeatability, particularly if both carrier phase and pseudorange data are used. Stochastic modeling may thus be an acceptible alternative, at least for the type of conditions encountered in this experiment. However, further analyses of stochastic estimation and WVR calibration are necessary to test the long-term validity of stochastic parameterization of the wet tropospheric delay at a given site. Results for the OVRO-Mojave baseline in southern California are not sensitive to the choice of tropospheric calibration scheme. Although the level of tropospheric fluctuations was comparable in southern California, the mean wet path delay at zenith was 3-5 times smaller than in the gulf. Thus for dry locations, WVRs may not be required to model accurately tropospheric effects on GPS baselines.

The high level of precision obtained in this first GPS experiment in the Gulf of California is encouraging in that the baselines of interest are located outside the U.S. fiducial network, in a region where wet tropospheric delays are significant. Expansion of the current network along the length of the gulf and into northern Baja California appears warranted and would help address problems related to the kinematics of the Pacific-North America plate boundary.

Acknowledgments. This research was carried out at the Jet Propulsion Laboratory, California Institute of Technology, under contract with the National Aeronautics and Space Administration and supported by The Geodynamics Program. We thank C. J. Vegos, S. J. Di Nardo, C. Gross, J. P. Dauphın, M. P. Golombek, and F. SuarezVidal for assistance with the field operations. All computations were conducted at the Jet Propulsion Laboratory using the GIPSY analysis software. We thank J. M. Davidson, R. N. Treuhaft, P. McFee, G. Blewitt, K. M. Larson, and the GIPSY analysis team for numerous contributions and discussions. We are very grateful to S. L. Lichten, R. W. King, and two anonymous reviewers for helpful comments. D.M.T. was supported in part by a National Research Council Fellowship.

\section{REFERENCES}

Bean, B. R., and E. J. Dutton, Radio Meteorology, National Bureau of Standards, U.S. Department of Commerce, Gaithersburg, Md., 1986.

Beckman, B., A water-vapor radiometer error model, IEEE Trans. Geosci. Remote Sens., GE-23, 474-478, 1985.

Bender, P. L., Present technology development needs for geodynamics in the 1990's, paper presented at the Fifth Session of the NASA Geodynamics Workshop, Warrenton, Va., 1983.

Berman, A. L., A new tropospheric range refraction model, in The Deep Space Network, Space Programs Summ. 37-65, vol. II, pp. 140-153, Jet Propulsion Laboratory, Pasadena, Calı., 1970.

Beutler, G., I. Bauersima, W. Gurtner, M. Rothacher, and T. Schildknecht, Evaluation of the 1984 Alaska Global Positioning System campaign with the Bernese GPS software, J. Geophys. Res., 92, 1295-1303, 1987.

Bierman, G. J., Factorization Methods for Discrete Sequential Estimatıon, 241 pp., Academic, Orlando, Fla., 1977.

Bock, Y., S. A. Gourevitch, C. C. Counselman III, R. W. King, and R. I. Abbot, Interferometric analysis of GPS phase observations, Manusc. Geod., 11, 282-288, $1986 a$.

Bock, Y., R. I. Abbot, C. C. Counselman III, and R. W. King, A demonstration of 1-2 parts in $10^{7}$ accuracy using GPS, Bull. Geod., $60,241-254,1986 b$.

Bossler, J. D., C. C. Goad, and P. L. Bender, Using the Global Positioning System (GPS) for geodetic positioning, Bull. Geod., 54, 553$563,1980$.

Chandrasekhar, S., Radiative Transfer, 393 pp., Oxford Universıty Press, New York, 1950. 
Chao, C. C., A new method to predict wet zenith range corrections from surface measurements, Tech. Rep. Calif. Inst. Technol. Jet Propul. Lab., 32-1256, XIV, 33-41, 1973.

Chao, C. C., The troposphere calibration model for Mariner Mars 1971, Tech. Rep. Calif. Inst. Technol. Jet Propul. Lab., 32-1587, 61-76, 1974.

Clafin, E. S., S. C. Wu, and G. M. Resch, Microwave radiometer measurement of water vapor path delay: Data reduction techniques, Deep Space Network Prog. Rep. 42-48, pp. 22-30, Jet Propul. Lab., Pasadena, Calif., 1978.

Coco, D. S., and J. R. Clynch, The variability of the tropospheric range correction due to water vapor fluctuations, in Proceedings of the Third International Geodetic Symposium on Satellite Doppler Positioning, vol. 1, pp. 475-495, New Mexico State University, Las Cruces, 1982.

Davidson, J. M., and D. W. Trask, Utilization of mobile VLBI for geodetic measurements, IEEE Trans. Geosci. Remote Sens., GE-23, $426-437,1985$.

Davidson, J. M., C. L. Thornton, C. J. Vegos, L. E. Young, and T. P. Yunck, The March 1985 demonstration of the fiducial network concept: A preliminary report, paper presented at the First International Conference on Precise Positioning with the Global Positioning System, Int. Assoc. of Geod., Rockville, April 15-19, 1985.

Davidson, J. M., et al., The March 1985 high precision baseline test of the JPL GPS-based geodetic system: A final report, JPL Publ., 87-35, 1987.

Davis, J. L., T. A. Herring, I. I. Shapiro, A. E. E. Rogers, and G. Elgered, Geodesy by radio interferometry: Effects of atmospheric modeling errors on estimated baseline length, Radio Sci., 20, 1593$1607,1985$.

Dixon, T. H., D. M. Tralli, and J. P. Dauphin, Geodetic baselines across the Gulf of California using the Global Positioning System, Gulf and Peninsular Provinces of the Californias, Mem. Am. Assoc. Pet. Geol., in press, 1988.

Dodson, A. H., Refraction and propagation delays in space geodesy, Int. J. Remote Sens., 7(4), 515-524, 1986.

Dravskikh, A. F., and M. A. Finkelstein, Tropospheric limitations in phase and frequency coordinate measurements in astronomy, Astrophys. Space Sci., 60, 251-265, 1979.

Evans, A. G., Comparison of GPS pseudorange and biased doppler range measurements to demonstrate signal multipath effects, paper presented at the Fourth International Geodetic Symposium on Satellite Positioning, Univ. of Tex. at Austin, 1986.

Gardner, C. S., Effects of horizontal refractivity gradients on the accuracy of laser ranging to satellites, Radio Sci., $11,1037-1044,1976$.

Gardner, C. S., J. R. Rowlett, and B. E. Hendrickson, Ray tracing evaluation of a technique for correcting the refraction errors in satellite tracking data, Appl. Opt., 17, 3143-3145, 1978.

Gary, B. L., S. J. Keihm, and M. A. Janssen, Optimum strategies and performance for the remote sensing of path-delay using groundbased microwave radiometers, IEEE Trans. Geosci. Remote Sens., $G E-23,479-484,1985$.

Gibbs, B. P., and V. Majer, Accuracy assessment of the atmospheric correction used in the NASA laser ranging program, Rep. BTS-FR81-166, 54 pp., Bus. and Technol. Syst., Inc., Seabrook, Md., 1981.

Gill, A. E., Atmosphere-Ocean Dynamics, Int. Geophys. Ser., vol. 30 edited by W. L. Donn, 662 pp., Academic, Orlando, Fla., 1982.

Hargrave, P. J., and L. J. Shaw, Large-scale tropospheric irregularities and their effect on radio astronomical seeing, Mon. Not. R. Astron. Soc., 182, 233-239, 1978.

Herring. T. A., Precision of vertical position estimates from very long baseline interferometry, J. Geophys. Res., 91, 9177-9182, 1986.

Hogg, D. C., F. O. Guiraud, and W. B. Sweezy, The short term temporal spectrum of precipitable water vapor, Science, 213,1112 1113, 1981.

Hopfield, H. S., Tropospheric range error at the zenith, report, Appl. Phys. Lab., Johns Hopkins Univ., Laurel, Md., June 1971.

Janssen, M. A., A new instrument for the determination of radio path delay due to atmospheric water vapor, IEEE Trans. Geosci. Remote Sens., GE-23, 485-490, 1985.

Kalman, R. E., A new approach to linear filtering and prediction problems, Trans. ASME, Ser. D, 32, 35-50, 1960.

Kroger, P. M., J. M. Davidson, and E. C. Gardner, Mobıle very long baseline interferometry and Global Positioning System measurement of vertical crustal motion, J. Geophys. Res., 91, 9169-9176, 1986.
Lanyi, G. E., Troposphertc delay effects in radio interferometry, Telecommun. Data Acquis. Prog. Rep. 42-78, pp. 152-159, Jet Propul. Lab., Pasadena, Calif, April-June 1984.

Lichten, S. M., and J. S. Border, Strategies for high precision Global Positionıng System orbit determination, J. Geophys. Res., 92 , 12,751-12,762, 1987.

Nahvi, M. J., M. Rahnemoon, and P. Hartl, Study of tropospheric water vapor modelling techniques for satellite microwave ranging final report, 224 pp., Eur. Space Agency, Univ. of Stuttgart, Stuttgart, Federal Republic of Germany, May 1986.

Reber, E. E., and J. R. Swope, On the correlation of the total precipitable water in a vertical column and absolute humidity, $J$. Appl. Meteorol., I1, 1322-1325, 1972.

Remondi, B. W., Global Positioning System carrier phase: Description and use, Bull. Geod., 59, 361-377, 1985.

Resch, G. M., Water vapor radiometry in geodetic applications, in Geodetic Refraction, edited by F. K. Brunner, pp. 53-84, SpringerVerlag, New York, 1984.

Resch, G. M., M. C. Chavez, and N. I. Yamane, Description and overview of an instrument designed to measure line-of-sight delay due to water vapor, Telecommun. Data Acquis. Prog. Rep. 42-72, pp 1-19, Jet Propul. Lab., Pasadena, Calif., 1982.

Resch, G. M., M. C. Chavez, N. I. Yamane, K. M. Barbier, and R. C. Chandlee, Water vapor radiometry research and development phase final report, JPL Publ., 85-14, 1985.

Saastamoinen, J., Atmospheric correction for the troposphere and stratosphere in radio ranging of satellites, in The Use of Artificial Satellites for Geodesy, Geophys. Monogr. Ser., vol. 15, edited by S. W. Henriksen, A. Mancini, and B. H. Chovitz, pp. 247-251, AGU, Washington, D. C., 1972.

Smith, E. K., Jr., and S. Weintraub, The constants in the equation for atmospheric refractive index at radio frequencies, Proc. IRE, 4I, 1035-1057, 1953.

Spilker, J. J., GPS signal structure and performance characteristics, $J$. Inst. Navig., 25(2), 121-146, 1978.

Tapley, B. D., J. B. Lundberg, and G. H. Born, The Seasat altimeter wet tropospheric range correction, J. Geophys. Res., 87, 3213-3220, 1982.

Thornton, C. L., and G. J. Bierman, $U D U^{T}$ covariance factorization for Kalman filtering in Control and Dynamic Systems, Adv. Theory Appl., vol. 16, edited by C. T. Leondes, pp. 177-248, Academic Orlando, Fla., 1980

Treuhaft, R. N., and G. E. Lanyi, The effects of the dynamic wet troposphere on radio interferometric measurements, Radio Sci., 22 , 251-265, 1987.

Walter, S. J., and P. L. Bender, Status of the slant path atmospheric refraction calibrator (SPARC) (abstract), Eos Trans. AGU, 68, 1239 1987.

Westwater, E. R. An analysis of the correction of range errors due to atmospheric refraction by microwave radiometric techniques, $T$ ech. Rep. IER 30-ITSA30, Environ. Sci. Serv. Admin., Boulder, Colo., 1967. (Available from Natl. Tech. Inform. Serv., Springfield, Va.)

Westwater, E. R., The accuracy of water vapor and cloud liquid determinations by dual-frequency ground based microwave radiometry, Radio Sci., 13, 677-685, 1978

Wu, S. C., Optimum frequencies of a passive microwave radiometer for tropospheric path length correction, IEEE Trans. Antennas Propag., $A P-27,233-239,1979$.

Wu, S. C., W. I. Bertiger, J. S. Border, S. M. Lichten, R. F. Sunseri, B. G. Williams, P. J. Wolf, and J. T. Wu, OASIS mathematical descriptıon, report, Jet Propul. Lab., Pasadena, Calif, 1986.

Yang, W. S., S. J. Franke, and G. W. Swenson, Jr., A system for measuring the "wet" component of atmospheric refraction on a trans-atmospheric radio link, interim report, Univ. of Ill., Urbana, July 1981 .

T. H. Dixon and D. M. Tralli, Jet Propulsion Laboratory, California Institute of Technology, MS 183-501, 4800 Oak Grove Drive, Pasadena, CA 91109

S. A. Stephens, Department of Astronomy, 1011 W. Springfield, University of Illinois, Urbana, IL 61801.

(Received July 13, 1987

revised January 7,1988 accepted November 18, 1987) 